\title{
A CONVERSE STEINHAUS THEOREM FOR LOCALLY COMPACT GROUPS
}

\section{S. M. SIMMONS}

ABSTRACT. Singular and absolutely continuous regular measures on locally compact groups are characterized in terms of the algebraic and topological structure of sets assigned positive measure.

A classical theorem of $\mathrm{H}$. Steinhaus asserts that if a set $E$ has positive left Haar measure, then the set $E E^{-1}$ contains a neighborhood of the identity. In this paper we prove a strong converse to this theorem, and so obtain a new characterization of absolutely continuous measures, which does not refer to Haar measure. We then give a related characterization of purely singular measures. The construction which underlies these results is carried out in the following.

Lemma. Let $\mu$ be a positive, finite, regular Borel measure on a locally compact group $G$, which is singular with respect to left Haar measure, $\lambda$, on $G$. Let $\mu$ be concentrated on a Borel set $R \subset G$ with $\lambda(R)=0$. Then for each compact set $K \subset R$, there is a Borel set $P \subset K$ such that $P P^{-1}$ has void interior and the complement of $P$ in $K$ is $\mu$-null.

Proof. Since the compact set $K$ is contained in a compactly-generated closed and open subgroup, we may suppose without loss of generality that $G$ is compactly generated. Since $\lambda(K)=0$, regularity of $\mu$ allows us to choose a sequence $U_{i}$ of open sets with $K \subset U_{i}$, for all $i=1,2,3, \cdots$, and $\operatorname{Lim} \lambda\left(U_{i}\right)=0$. We also choose symmetric neighborhoods $W_{i}$ of the identity $e$, with $K W_{i} \subset U_{i}$ for all $i$. Because of the metrization theorem for groups [1], there exists a compact normal subgroup $N$ of $G$ such that $G / N$ is separable and metric, and with $N \subset \bigcap_{i=1}^{\infty} W_{i}$, so that $K N \subset K W_{i} \subset U_{i}$ for all $i$, and consequently, $\lambda(K N)=0$. Now let $Z=\{x \in G: \mu(x K N)=0\}$. We claim that the cosets $\{x N: x \in Z\}$ are dense in $G / N$. For consider an arbitrary nonvoid open set $V \subset G$ and the corresponding open set $\{v N: v \in V\}$.

Presented to the Society, April 22, 1972; received by the editors March 4, 1974. AMS (MOS) subject classifications (1970). Primary 43A05, 28A70.

Key words and phrases. Absolute continuity, regularity of measures. 
Letting $\xi_{E}$ denote the characteristic function of a set $E \subset G$, and using Fubini's theorem, we compute that

$$
\begin{aligned}
\int_{V N} \mu(x K N) d \lambda(x) & =\int \xi_{V N}(x) \int \xi_{x K N}(y) d \mu(y) d \lambda(x) \\
& =\iint \xi_{V N}(x) \xi_{(K N)^{-1}}\left(y^{-1} x\right) d \lambda(x) d \mu(y) \\
& =\iint \xi_{V N}(y x) \xi_{(K N)^{-1}}(x) d \lambda(x) d \mu(y) \\
& =\int \xi_{(K N)^{-1}}(x) \int \xi_{V N}(y x) d \mu(y) d \lambda(x)=0,
\end{aligned}
$$

since $\lambda\left((K N)^{-1}\right)=\lambda(K N)=0$. Thus the original integrand, $\mu(x K N)$, will vanish for $\lambda$-almost all $x \in V N$. Taking any $x \in V N$ with $\mu(x K N)=0$, it follows that $x N \in\{v N: v \in V\}$, so the claim is established.

Now consider the set

$$
Z_{1}=\{x \in G: \mu(K N \cap x K N)=0\} .
$$

Since $\mu(K N \cap x K N) \leq \mu(x K N)$, we have that $Z \subset Z_{1}$, so that the set $\{x N$ : $\left.x \in Z_{1}\right\}$ is dense in $G / N$. Using separability of $G / N$, choose a countable set $D \subset Z_{1}$ such that $\{d N: d \in D\}$ is dense in $G / N$. Now let

$$
S=\bigcup\{K N \cap d K N: d \in D\}
$$

and note that $S \subset K N$ and that

$$
\mu(S) \leq \sum_{d \in D} \mu(K N \cap d K N)=0 .
$$

Finally, let $B=K N \sim S$, the complement of $S$ in $K N$. We will show that $B B^{-1}$ has void interior. For, if there exists a nonvoid open set $V \subset B B^{-1}$, then by the choice of $D$, there is a $d \in D$ with $d N \in\{v N: v \in V\}$. Thus $d N C$ $V N \subset B B^{-1} N$, and so there exist $k_{1} n_{1} \in B$ and $k_{2} n_{2} \in B$ and $n_{0}, n_{3} \in N$ such that $d n_{0}=k_{1} n_{1}\left(k_{2} n_{2}\right)^{-1} n_{3}$, so, using normality of $N$,

$$
k_{1} n_{1}=d n_{0} n_{3}^{-1} k_{2} n_{2} \in d N N^{-1} K N=d K N \text {. }
$$

But this means $k_{1} n_{1} \in K N \cap d K N \subset S$, a contradiction, since $S$ is disjoint from $B$, and $k_{1} n_{1}$ was chosen from $B$. The contradiction shows $B B^{-1}$ has void interior. Moreover, since $K \subset K N$, we have $K \sim S \subset K N \sim S=B$, and the inclusion

$$
(K \sim S)(K \sim S)^{-1} C B \cdot B^{-1}
$$


shows that the interior of $(K \sim S)(K \sim S)^{-1}$ must also be void.

We may now take $P=K \sim S$ to establish the Lemma, noting that the complement of $P$ in $K$ is the set $K \cap S$, which is $\mu$-null since $S$ is $\mu$-null.

It is now possible to derive the two characterization theorems.

Theorem 1. Let $\mu$ be a complex regular Borel measure on $G$, with total variation $|\mu|$. The following statements are equivalent:

(1) For each compact $E$ in $G$ with $|\mu|(E)>0$, the set $E E^{-1}$ has nonvoid interior.

(2) For each compact set $E$ in $G$ with $|\mu|(E)>0$, the set $E E^{-1}$ contains a neighborhood of the identity.

(3) For each pair $A, B$ of compact sets with $|\mu|(A)>0$ and $|\mu|(B)>$ 0 , the set $A B^{-1}$ has nonvoid interior.

(4) The measure $\mu$ is absolutely continuous with respect to left Haar measure $\lambda$ on $G$.

Proof. Statements (3) and (2) clearly imply (1), and that (4) implies (3) and (2) is the content of the classic Steinhaus theorem [1, Theorem 20.17]. To establish the equivalence of all four statements, we need only show that (1) implies (4). So suppose that $\mu$ satisfies the hypothesis of (1), and let $\mu=\sigma+' \eta$ be the Lebesgue decomposition of $\mu$, where $\sigma$ is absolutely continuous with respect to $\lambda$, and $\eta$ is singular with respect to $\lambda$. We will prove (4) by show ing that $\eta=0$. Since statement (4) is known to imply (2), and consequently (1), the measure $\sigma$ satisfies the hypothesis of (1) as does $\mu$. It follows easily that the difference, $\eta=\mu-\sigma$, also satisfies the hypothesis of (1). Suppose, for a contradiction, that $\eta \neq 0$. Then $|\eta|$ is a nontrivial measure, concentrated on a Borel set $R$ with $\lambda(R)=0$. By regularity of $|\eta|$, we may choose a compact set $K_{1} \subset R$ with $|\eta|\left(K_{1}\right)>0$. The Lemma above then guarantees the existence of a Borel set $P \subset K_{1}$ with $|\eta|(P)=|\eta|\left(K_{1}\right)$, and such that $P \cdot P^{-1}$ has void interior. Again by regularity of $|\eta|$, there is a compact set $K \subset P$ with $|\eta|(K)>0$. But this contradicts the fact that $\eta$ satisfies the hypothesis of (1), since $K K^{-1} \subset P P^{-1}$ implies that $K K^{-1}$ has void interior. The contradiction shows that $\eta=0$, and the proof is complete.

Our next theorem shows that singular measures have antithetical properties to those in Theorem 1.

Theorem 2. A regular measure $\mu$ on $G$ is singular with respect to Haar measure $\lambda$, if and only if $\mu$ is concentrated on a $\sigma$-compact set $B$ such that $B B^{-1}$ has void interior. 
Proof. For the "if" statement, let $\mu=\sigma+\eta$ be the Lebesgue decomposition of $\mu$, as above. Since $\mu$ is concentrated on $B$, so is $\sigma$, but by the property of $B$ in the hypothesis and Theorem 1 above, it follows that $|\sigma|(B)$ $=0$, so that $\sigma=0$. Hence $\mu=\eta$, so that $\mu$ is singular.

For the "only if" part, we may suppose without loss of generality that $\mu$ is nonnegative and concentrated on $E$ with $\lambda(E)=0$. The conclusion will follow from the construction of a sequence $\left\{F_{i}\right\}$ of compact sets with the properties:

(1) $F_{1} \subset F_{2} \subset F_{3} \subset \ldots \subset \subset$.

(2) For each $i, F_{i} F_{i}^{-1}$ has void interior.

(3) $\operatorname{Lim} \mu\left(F_{i}\right)=\mu(E)$.

This sequence is formed as follows: Using regularity of $\mu$ we choose a sequence $K_{i}$ of compact sets so that $K_{1} \subset K_{2} \subset K_{3} \subset \ldots \subset E$ and $\operatorname{Lim} \mu\left(K_{i}\right)=\mu(E)$. By the Lemma above, we decompose each $K_{i}$ into $K_{i}=$ $P_{i} \cup N_{i}$ where $P_{i} P_{i}^{-1}$ has void interior and $\mu\left(N_{i}\right)=0$. Let $N=\bigcup_{i=1}^{\infty} N_{i}$, so that $K_{1} \sim N \subset K_{2} \sim N \subset \ldots \subset E$ and $\mu\left(K_{i} \sim N\right)=\mu\left(K_{i}\right)$, for all $i$. Again using regularity of $\mu$, we let $F_{0}=\varnothing$ and choose by induction a sequence $\left\{F_{i}\right\}$ of compact sets such that $F_{i-1} \subset F_{i} \subset K_{i} \sim N$, and

$$
\left|\mu\left(K_{i} \sim N\right)-\mu\left(F_{i}\right)\right|=\left|\mu\left(K_{i}\right)-\mu\left(F_{i}\right)\right| \leq 1 / i .
$$

This being done, properties (1) and (3) are evident, and (2) follows from the inclusion $F_{i} \subset K_{i} \sim N \subset K_{i} \sim N_{i} \subset P_{i}$, so that $F_{i} F_{i}^{-1} \subset P_{i} P_{i}^{-1}$; the choice of $P_{i}$ insures that $F_{i} F_{i}^{-1}$ has void interior.

To obtain the theorem, we let $B=\bigcup_{i=1}^{\infty} F_{i}$. Clearly $B \subset E$ with $\mu(E \sim B)=0$, so that $\mu$ is concentrated on $B$. To see that $B B^{-1}$ has void interior we briefly suppose the contrary, and note that because of property (1), $B B^{-1} \subset \bigcup_{i=1}^{\infty} F_{i} F_{i}^{-1}$. The Baire category theorem then asserts that one of the compact sets $F_{i} F_{i}^{-1}$ has nonvoid interior, a contradiction of property (2) of the sequence $\left\{F_{i}\right\}$. Since $B$ is $\sigma$-compact by construction, $B$ has the properties claimed in the statement of the theorem.

\section{REFERENCES}

1. E. Hewitt and K. A. Ross, Abstract harmonic analysis. Vol 1: Structure of topological groups. Integration theory, group representations, Die Grundlehren der math. Wissenschaften, Band 115, Academic Press, New York; Springer-Verlag, Berlin, 1963. MR 28 \# 158.

DEPARTMENT OF MATHEMATICS, EASTERN WASHINGTON STATE COLLEGE, CHENEY, WASHINGTON 99004 